\title{
Enfermedad de Castleman localizada: descripción de un caso y revisión de la literatura
}

\author{
I. COCA PRIETO, M. V. ORTEGA JIMÉNEZ', E. FERNÁNDEZ RUIZ², J. C. GAVILÁN \\ CARRASCO, F. BERMÚDEZ RECIO
}

Servicio de Medicina Interna, 'Anatomía Patológica y ${ }^{2}$ Otorrinolaringología. Hospital

Universitario Virgen de la Victoria. Málaga

\author{
LOCALIZED CASTLEMAN'S DISEASE: DESCRIPTION OF A CASE \\ AND REVIEW OF THE LITERATURE
}

\begin{abstract}
RESUMEN
La enfermedad de Castleman (EC) es una entidad rara definida por sus hallazgos anatomopatológicos: hiperplasia de las células dendríticas de los ganglios linfáticos y marcada proliferación capilar. Se han descrito dos tipos histológicos: la variedad hialinovascular y la plasmocelular. Desde el punto de vista clínico se han identificado dos cuadros bien diferenciados: la forma localizada (ECL), de curso clínico benigno, y la forma multicéntrica (ECM) de peor pronóstico. Presentamos un caso de EC localizada en la región cervical, en la que la resección quirúrgica de la adenopatía resultó ser diagnóstica y terapéutica. La variedad histológica fue del tipo hialinovascular.
\end{abstract}

PALABRAS CLAVE: Hiperplasia angiofolicular linfoide. Hiperplasia nodular gigante. Enfermedades linfáticas

\begin{abstract}
Castleman's disease is a rare entity which is caracterized by its histological features: hyperplasia of lymph nodes and capillary proliferation. Two histological patterns has been described: hyaline vascular type and plasma cell type. From a clinical viewpoint has been identified two different clinical course: a localized type (ECL) usually of benign clinical course and a multicentric type (ECM) of worst prognosis. We present a case of Castleman's disease localized in the neck region in which the excision was both diagnostic and therapeutic. The variety histological was hyaline-vascular type.
\end{abstract}

KEY WORDS: Angiofollicular lymph node hyperplasia. Giant lymph node. Lymphatic diseases.

Coca Prieto I, Ortega Jiménez MV, Fernández Ruiz E, Gavilán Carrasco JC, Bermúdez Recio F. Enfermedad de Castleman localizada: descripción de un caso y revisión de la literatura. An Med Interna (Madrid) 2003; 20: 534-536.

\section{INTRODUCCIÓN}

La enfermedad de Castleman (EC), también llamada hiperplasia nodular gigante, hiperplasia angiofolicular linfoide, es una entidad infrecuente, fue descrita por primera vez en 1956 , en un paciente que presentaba una masa en la región mediastínica (1). Posteriormente fueron comunicados casos en otros territorios ganglionares y extraganglionares. Existen dos variantes histológicas (2): la hialinovascular (HV), que acontece en el $80-90 \%$ de los casos $(2,6)$, y que habitualmente es asintomática; y la plasmocelular (PC), el 10\% de los casos, y que suele presentarse con afectación del estado general, anemia, hipergammaglobulinemia y hepatoesplenomegalia. Existen dos formas clínicas de presentación (2), la localizada (ECL) o clásica, y la forma multicéntrica (ECM) $(3,10)$. Presentamos un caso de ECL, limitado al cuello, perteneciente a la variedad histológica hialino vascular, y que como en la mayoría de los casos publicados, la exéresis de la adenopatía fue diagnóstica y terapéutica.

\section{CASO APORTADO}

Mujer de 27 años, fumadora de 20cigarrillos/día, sin otros antecedentes de interés, que consulta en nuestro Servicio por una tumoración laterocervical izquierda de un mes y medio de evolución, no dolorosa, de aparición brusca y crecimiento lento. No refería fiebre, disfonía ni odinofagia. A la exploración física presentaba una adenopatía laterocervical izquierda móvil de 5 x 5 x 4cm, no dolorosa, bien delimitada, aunque adherida a plano muscular superficial y fascia. El resto de la exploración general y ORL no presentaba otras alteraciones.

Las pruebas complementarias con sistemático de sangre, VSG, extensión de sangre periférica, bioquímica, proteinograma e inmunoglobulinas fueron normales. Asimismo las cifras de valores de TSH, C3, C4, PCR y marcadores tumorales estaban dentro de rango normal. El Mantoux fue negativo. El estudio serológico mostraba Ac de clase $\mathrm{IgG}$ para toxoplasma, citomegalovirus y virus de Epstein Barr con Ac de clase IgM negativo para todos ellos, así como Ac frente a VIH negativos. La radiografía de tórax fue normal. La ecografía cervical mostraba un nódulo sólido muy vascularizado de $3,5 \mathrm{~cm}$, situado

Trabajo aceptado: 10 de febrero de 2003

Correspondencia: I. Coca Prieto. C/ Concejal Peña Abizanda, 1-6º F. 29010 Málaga. e-mail inmacopri@ hotmail.com 
debajo del músculo esternocleidomastoideo, de borde liso ligeramente ondulado y bien definido con ecogenicidad heterogénea con septos lineales y microfocos hiperecogénicos en su polo inferior. A su alrededor, numerosos nódulos sólidos ovoideos, el mayor de $2 \mathrm{~cm}$ no vascularizados, concordantes con adenopatías. Las glándulas parótida y submandibulares eran normales. La TAC cérvico/torácica/abdominal mostraba nódulo sólido de aproximadamente 4cm de diámetro mayor, en la cadena laterocervical izquierda, hiperdenso tras la inyección de contraste con pequeñas linfadenopatías satélites menores de $1 \mathrm{~cm}$ (Fig. 1). El tórax, abdomen y la pelvis fueron normales. Se realizó una PAAF bajo control de TAC que mostró linfadenitis reactiva inespecífica, por lo que se procedió a la extirpación de la adenopatía de mayor tamaño, cuya anatomía patológica mostró hiperplasia angiolinfoide del tipo histológico hialinavascular (Fig. 2).

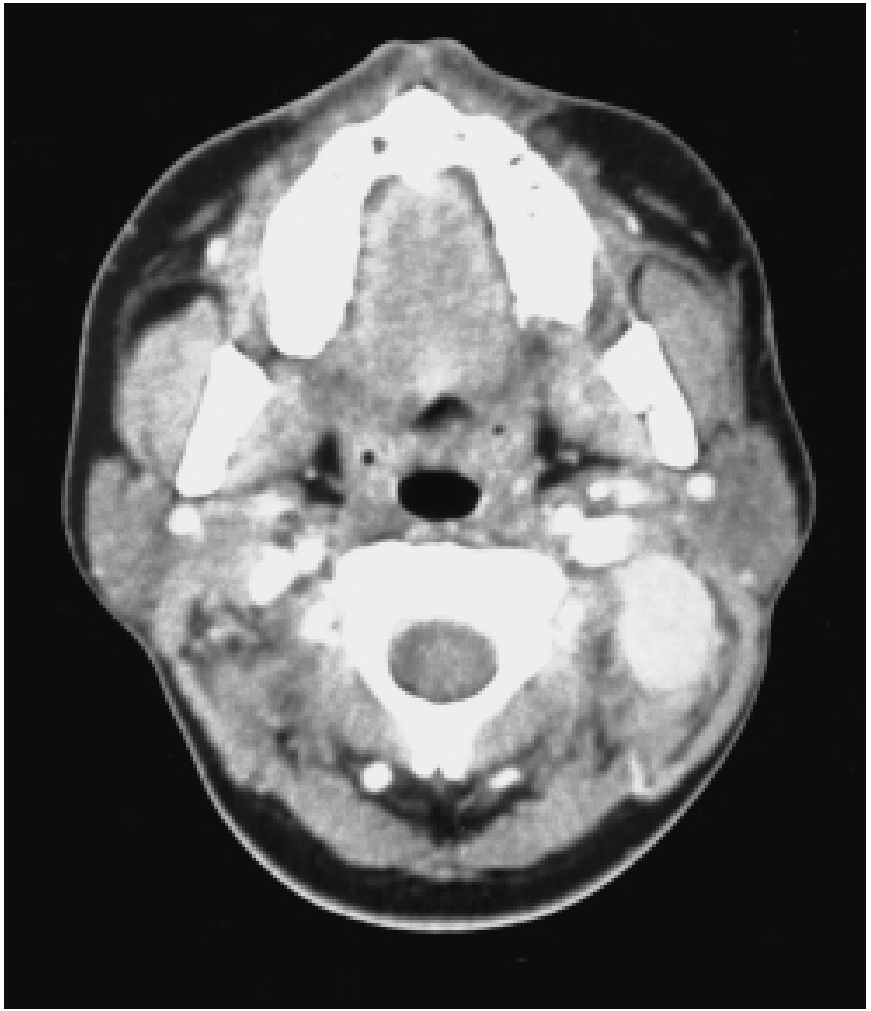

Fig. 1. NóПdulo sólido hiperdenso a la derecha de la imagen.

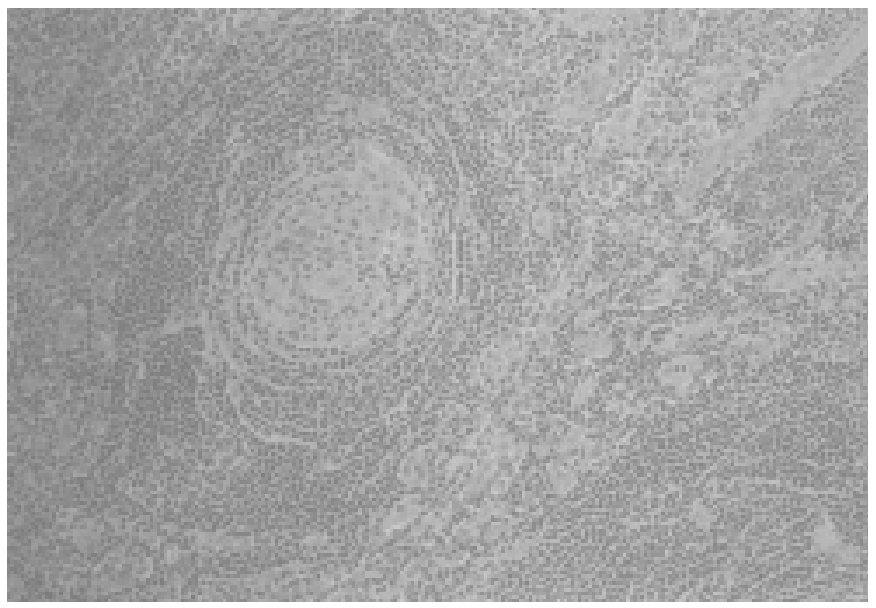

Fig. 2. EC tipo histológico hialinovascular.
Tras un año de la intervención, la paciente se encuentra asintomática en el seguimiento, con pruebas complementarias de analítica de sangre, proteinograma, inmunoglobulinas y estudio radiológico de control normales.

\section{DISCUSIÓN}

La EC como ya hemos comentado tiene dos formas clínicas de presentación: la forma localizada (ECL), más frecuente del tipo histológico hialinovascular $(90 \%)$ y el tipo plasmocelular; se manifiesta en adultos jóvenes de 15 a 30 años, con igual prevalencia de sexo y se presenta como una masa única asintomática. En el 50\% de los casos de la variedad plasmocelular existen síntomas constitucionales acompañantes como fatiga, fiebre, sudoración, hipoalbuminemia, leucocitosis, anemia y aumento de la velocidad de sedimentación (2). La localización más frecuente afectada es el mediastino (60\%); le siguen en orden de frecuencia, el cuello (14\%), el abdomen (11\%), retroperitoneo (11\%), y la axila $(4 \%)$, (4). En la localización cervical es común la afectación de los ganglios cervicales situados bajo el esternocleidomastoideo, como en nuestro caso $(3,8)$; menos frecuentes son las presentaciones en la parótida, el suelo de la boca, glándula submandibular, la laringe, el paladar y el espacio parafaríngeo $(5,6)$

La forma multicéntrica (ECM), suele ser del tipo histológico plasmocelular $(2,3)$ se manifiesta en personas de mayor edad, aproximadamente de 50 años, con una relación 2,5:1 hombre: mujer, y la clínica es un síndrome constitucional con linfadenopatía generalizada, anemia y hepatoesplenomegalia; el pronóstico en estos casos, es menos favorable ya que hasta en un $32 \%$ de los pacientes se han desarrollado neoplasias, particularmente sarcomas de Kaposi, linfomas no Hodking y plasmocitomas. No obstante, se han descrito casos de neoplasias asociadas a las formas localizadas $(3,10,12)$.

La patogénesis de la enfermedad es desconocida aunque se ha relacionado con factores causales como la inflamación crónica, los estados de inmunodeficiencia, los fenómenos de autoinmunidad y, recientemente la interleucina-6 y otras interleucinas, que explicarían el desarrollo de linfomas malignos $(7,9,10)$.También, en los últimos años se ha considerado al Herpes-virus tipo 8 en la etiología de la enfermedad multicéntrica (13).

La histología del tipo hialinovascular está caracterizada por la proliferación de folículos linfoides. Estos folículos están formados por centros germinales rodeados de linfocitos maduros dispuestos en capas concéntricas (piel de cebolla) con capilares que se introducen en los centros germinales, presentan depósitos de hialina conectados por un estroma vascular prominente y con escasas células plasmáticas. Los estudios inmunohistológicos muestran un patrón policlonal de linfocitos B y de células plasmáticas. En la variedad plasmocelular los folículos linfoides son mayores y los centros germinales más prominentes. La zona interfolicular es menos vascular y presenta abundantes células plasmáticas. La inmunohistología muestra expresión policlonal de células plasmáticas, aunque se ha descrito expresión monoclonal de éstas (14).

El diagnóstico definitivo se basa en criterios clínicos e histológicos. Las pruebas de laboratorio, las técnicas de imagen y la punción aspiración con aguja fina nos pueden ofrecer información y descartar otros procesos (8). 
El diagnóstico diferencial de la enfermedad localizada se realizará con otras causas más comunes de masa cervical solitaria (Tabla I) $(5,6)$.

\section{TABLA I}

\section{DIAGNÓSTICO DIFERENCIAL DE MASA CERVICAL SOLITARIA}

Procesos inflamatorio-infecciosos

Linfadenitis, tuberculosis, sarcoidosis, toxoplasmosis, citomegalovirus, mononucleosis, enfermedad por arañazo de gato, infección por el $\mathrm{VIH}$

Neoplasia de cabeza y cuello

Lipoma, linfoma, sarcoma, paraganglioma, schwanoma, nódulos metastásicos

Tumoraciones congénitas
Teratoma, quiste dermoide, quiste branquial, linfangioma, quiste tirogloso, hemangioma

\section{Bibliografía}

1. Castleman B, Iverson L, Pardo Mendedes V. Localized mediastinal lymph node hyperplasia resembling thymoma. Cancer 1956; 9:822830.

2. Keller AR, Hochholzer L, Castleman B. Hyalinevascular and plasma cell types of giant lymph node hyperplasia of mediastinum and other locations. Cancer 1972; 29: 670-683.

3. Sanz García RS, Guerra Vales JM, De Prada I, Martínez MA, Guillén Camargo V. Enfermedad de Castleman localizada asociada a linfoma de alto grado. An Med Interna (Madrid) 1999; 16: 305307

4. Gangopadhyay K, Mahasin ZZ, Kfoury H. Castleman disease (giant lymph node hyperplasia). Arch Otolaryngol Head Neck Surg 1997; 123: 1137.

5. Yi AY, de Tar M, Becker TS, Rice DH. Los Angeles, California. Giant lymph node hyperplasia of the head and neck (Castleman's disease): A report of five cases. Otolaryngol Head Neck Surg 1995; 113: 462-6.

6. Goodisson DW, Carr RJ, Stirling RW: Parotid presentation of Castleman's disease: report of a case. J Oral Maxillofac Surg 1997; 55: 515.

7. Leger-Ravet N, Peuchmaur M, Devergne $\mathrm{O}$ et al. Interleukin 6 gene expression un Castleman disease. Blood 1991; 78: 2923-2930.

8. Frizera G, Peterson BA, Bayrd ED, Goldam A. A sistematic lymp-
El tratamiento de la forma localizada es la resección quirúrgica lo más radical posible, tanto en el tipo hialinovascular como el tipo plasmocelular; con una curación de casi el 100\% de los casos y con excepcionales recurrencias (10-12). Otras terapias como la resección parcial y la radioterapia, se reservan para los pacientes con lesiones no resecables. En la forma multicéntrica, es difícil hablar del tratamiento ideal, dado el escaso número de pacientes de las series publicadas y la amplia variabilidad de los resultados descritos en la literatura. Se han utilizado tratamientos muy diversos como los esteroides en monoterapia o conjuntamente con agentes quimioterápicos (ciclofosfamida, vincristina, clorambucilo), sin evidencia de eficacia de ninguno de ellos, y con respuestas clínicas muy variadas $(10,11)$.

El seguimiento a largo plazo y la vigilancia periódica aún no están definidos para ninguna de las formas clínicas, aunque tal vez sea lo más prudente para detectar recurrencias de la enfermedad o el desarrollo de enfermedades malignas. hoproliferative disorder with morphologic features of Castleman disease; Clinical findings and clinicopathologic correlation in 15 patients. J Clin Oncol 1985; 3: 1202-1216.

9. Arranz R, Montesinos M, López Botet M, Acevedo A, Fernández Rañada JM. Enfermedad de Castleman: descripción y comentarios a propósito de 3 casos. Med Clin (Barc) 1994; 103: 620-622.

10. Herrada J, Cabanillas F, Rice L, Manning J, Pugh W. The clinical behavior of localized and multicentric Castleman disease. Ann Intern Med 1998; 120: 657-662.

11. Chronowski GM, HA ChS, Wilder RB, Cabanillas F, Manning J, Cox JD. Treatment of unicentric and multicentric Castleman disease and the role of radiotherapy. Cancer 2001; 92: 670-6.

12. Bowne WB, Lewis JJ, Filippa DA, Nieswizky R, Brooks AD, Burt $\mathrm{ME}$, et al. The management of unicentric and multicentric Castleman's disease. Cancer 1999; 85: 706-17.

13. Scot C. Remick, Madhumita Patnaik, Navid M.Ziran, Karsten R Liegmann, et al. Human Herpesvirus-8- associated disseminated angiosarcoma in an HIV-seronegative woman: Report of a case and limite case-control virologic study in vascular tumors. Am J Med 2000; 108: 660-664.

14. Shahidi H, Myers JL, Kvale PA. Castlema'n disease. Mayo Clin Proc. 1995 ; 70: 969-977. 